\title{
An Improved Scaling Procedure for Analysis and Simplification of Process Models
}

\author{
Sudhakar Munusamy ${ }^{\mathrm{a}}$, Parham Mobed ${ }^{\mathrm{b}}$, Debangsu Bhattacharyya ${ }^{\mathrm{c}}$, \\ Raghunathan Rengaswamyd,* \\ ${ }^{a}$ Department of Chemical Engineering, Texas Tech University, Lubbock Texas, \\ USA.Currently with Gyan Data Private Limited, Chennai, India. \\ ${ }^{b}$ Department of Chemical Engineering, Texas Tech University, Lubbock Texas, USA. \\ ${ }^{c}$ Department of Chemical Engineering, West Virginia University, Morgantown, West \\ Virginia, USA. \\ ${ }^{d}$ Department of Chemical Engineering, Indian Institute of Technology Madras, Chennai \\ 600036, India. Adjunct Professor at Department of Chemical Engineering, Texas Tech \\ University, Lubbock, Texas, USA.
}

\begin{abstract}
Systematic scaling analysis of model equations can be valuable as a tool for developing computationally tractable simulations of physical systems. The scaling analysis methods in literature pose difficulties in the calculation of scale and reference values, when nonlinear terms are involved in the model equations. Further, existing methods involve trial and error procedures in the scaling process. In this paper, a systematic approach for handling nonlinear terms is suggested, which results in appropriate scale and reference values that render the dimensionless variable variations to be of order one. Further, trial and error procedures are avoided through a new approach wherein a set of nonlinear algebraic equations are solved to identify the scale and reference values. The proposed scaling approach is common to any given model equations with fixed parameters. However, it is to be noted that the proposed procedure may not handle situations when model
\end{abstract}

\footnotetext{
*Corresponding author: Email: raghur@iitm.ac.in
} 
equations exhibit steady state multiplicity and have dynamic multi-mode regimes. The proposed scaling procedure is illustrated through various examples of different complexities. A 1D model of WGS reactor as a case study shows the effectiveness of the obtained scale and reference values in obtaining simplified model which represents the steady state and dynamic variations of the variables.

Keywords: Scaling analysis, Model simplification, Water gas shift reactor

\section{Introduction}

Scaling analysis is a systematic approach that can be used to identify phenomena occurring at various scales. This information can be used to simplify a given set of equations by neglecting phenomena, which occur at scales that are different from the scale of interest. In this approach, a given set of equations is made dimensionless, resulting in several dimensionless groups of varying magnitudes. These dimensionless groups represent the relative effects of phenomena or mechanisms and therefore help identify dominant phenomena/mechanisms in the scale of interest. A number of authors have used scaling analysis for model simplification and identification. For example, Dahl et al. (2004) have used scaling analysis to get insights into the behavior of fluid aerosol reactor without performing actual simulations. Kopaygorodsky et al. (2004) have used scaling analysis to identify key differences between the modeling assumptions for conventional pressure swing adsorption and ultra-rapid pressure swing adsorption. Kaisare et al. (2005) have used scaling analysis to identify phenomena occurring at varying scales in a reverse flow reactor. Balaji et al. (2008) have used scaling analysis for reverse flow reactor and have shown ways of simplifying the model equations. Rao et al. (2010) have used scaling analysis for pulsed pressure swing 
adsorber to identify useful correlations in terms of dimensionless numbers. Rezvanpour et al. (2012) have studied electro-hydrodynamic atomization process using scaling analysis to simply the model and to find a correlation relating efficiency with a single dimensionless number involving the parameters of the process. Baldea and Daoutidis (2007) have used scaling analysis for auto-thermal reactors to identify a non-stiff model by separating fast and slow time scales. Krantz (2007) have described the method of scaling analysis in a book for various transport and reaction process.

There are two important gaps in all these works that use scaling analysis. In the scaling methodology described by Krantz (2007), all dependent and independent variables in the equations are made dimensionless by choosing appropriate scale(s) and reference factor(s). This results in a minimum parametric representation of the model equations. Thus the solution of these equations can be expressed in terms of dimensionless groups. The form of these dimensionless groups and the methods used for obtaining scales usually involve trial and error methods. In Krantz et al. (2012), it is mentioned that one has to know the controlling mechanism while forming a unique dimensionless equation which varies in the order of 1 . Identifying this controlling mechanism is not obvious and in most cases this usually involves a trial and error process. Further, in all these works on scaling analysis, the scaling of nonlinear terms in the equations is addressed in an empirical manner. The scale for nonlinear terms are usually taken to be some characteristic maximum (Balaji et al., 2008; Krantz, 2007), but obtaining this maximum is not obvious without simulating the corresponding equations. We address these gaps in the literature by: (i) proposing an approach that avoids the trialand-error method for deriving scales, and (ii) we focus on nonlinear terms and suggest a systematic way to obtain appropriate scales for these terms. 
The proposed method for scaling analysis is general and straight-forward to apply to any given set of equations. The proposed method is described in several steps and explained though examples of varying complexity. We apply the techniques developed in this paper and calculate scale and reference values for a 1D model of water gas shift (WGS) reactor system, which involves complex nonlinear terms and differential algebraic equations. The obtained scale and reference values are shown to be appropriate in making the corresponding dimensionless variables to vary in the order of 1 . Analysis of obtained scale and reference values through relevant dimensionless groups results in a simplified model. The performance of the simplified model based on these scales is evaluated by comparing the simulation results with a detailed model and bench-marking the respective computational performances.

\section{Model simplification using current method of scaling analysis}

Systematic scaling analysis of model equations can identify phenomena with varying importance thereby providing a rational approach for model simplification through elimination of terms and elimination of equations with minimal impact on the simulation results. Scaling analysis involves identifying appropriate scale and reference values to make the entire dependent and independent variables in a model to be dimensionless and vary in the order of 1, i.e. these dimensionless variables vary from zero to near one. This type of representation for a model is termed as minimum parametric representation. In this representation, it is easy to identify terms (which corresponds to some physical phenomenon) of least importance and one can discard them to obtain a simplified model. 
In this section we first provide a general description of the scaling analysis and point out the deficiencies in the existing procedures at appropriate places. The method of scaling analysis followed in the literature is described below through several steps using a simple example.

Step 1: Consider the following system of equations

$$
\begin{aligned}
& \frac{d y_{1}}{d x}=f_{11}\left(y_{1}, y_{2}, x\right)+f_{12}\left(y_{1}, y_{2}, x\right) \\
& \frac{d y_{2}}{d x}=f_{21}\left(y_{1}, y_{2}, x\right)+f_{22}\left(y_{1}, y_{2}, x\right)
\end{aligned}
$$

The initial conditions for the above equations are given by

$$
y_{1}(x=0)=y_{10} ; \quad y_{2}(x=0)=y_{20}
$$

Step 2: Define dimensionless quantities (involving scale and reference values) for dependent and independent variables and introduce them into the equations

$$
y_{1}^{*} \equiv \frac{\left(y_{1}-y_{1, r}\right)}{y_{1, s}} ; \quad y_{2}^{*} \equiv \frac{\left(y_{2}-y_{2, r}\right)}{y_{2, s}} ; \quad x^{*} \equiv \frac{x}{x_{s}}
$$

In the above definition, variables with subscript 's' and ' $r$ ' represent scale and reference values respectively and variables with superscript '*' represent dimensionless quantities. Reference and scale values are introduced so that resulting dimensionless variable starts from zero and vary in order of 1 respectively. This means reference value is required only for variables which are not starting from zero. Hence in the above example, reference value is not introduced in the definition for dimensionless independent variable. However there are situations (for example, for fluid flow problem involving annulus pipe) where independent variables does not start from zero and in those situations one need to have reference values. Introducing these defini- 
tions in Eq. (1), Eq. (2) and Eq. (3)

$$
\begin{aligned}
& \frac{y_{1, s}}{x_{s}} \frac{d y_{1}^{*}}{d x^{*}}=f_{11}\left(y_{1}^{*} y_{1, s}+y_{1, r}, y_{2}^{*} y_{2, s}+y_{2, r}, x^{*} x_{s}\right)+f_{12}\left(y_{1}^{*} y_{1, s}+y_{1, r}, y_{2}^{*} y_{2, s}+y_{2, r}, x^{*} x_{s}\right) \\
& \frac{y_{2, s}}{x_{s}} \frac{d y_{2}^{*}}{d x^{*}}=f_{21}\left(y_{1}^{*} y_{1, s}+y_{1, r}, y_{2}^{*} y_{2, s}+y_{2, r}, x^{*} x_{s}\right)+f_{22}\left(y_{1}^{*} y_{1, s}+y_{1, r}, y_{2}^{*} y_{2, s}+y_{2, r}, x^{*} x_{s}\right)
\end{aligned}
$$

$$
y_{1}^{*}\left(x^{*} x_{s}=0\right)=\frac{\left(y_{10}-y_{1, r}\right)}{y_{1, s}} ; \quad y_{2}^{*}\left(x^{*} x_{s}=0\right)=\frac{\left(y_{20}-y_{2, r}\right)}{y_{2, s}}
$$

In the above equations, $\left(f_{11}, f_{12}, f_{21}, f_{22}\right)$ can represent linear or nonlinear terms involving dependent and independent variables. For these terms, once appropriate dimensionless variable definitions are introduced, one should be able to separate them into terms involving only scale and reference values and terms involving only dimensionless variables. For example, after scaling the term $f_{11}$, this needs to be written as a product of two terms $f_{11, s}=f_{11}\left(y_{1, s}, y_{1, r}, y_{2, s}, y_{2, r}, x_{s}, x_{r}\right)$ and $f_{11}^{*}=f_{11}\left(y_{1}^{*}, y_{2}^{*}, x^{*}\right)$. This separation becomes difficult for most of the nonlinear terms and there is hardly any work in the open literature that provides a rational approach to handling them. For example, consider the nonlinear first order kinetic term

$$
r_{A}=k_{0} e^{-\frac{E_{a}}{R T}} C_{A}
$$

For the above nonlinear term, on introducing the dimensionless variable definition for the variables $C_{A}$ and $T$, it becomes

$$
r_{A}=k_{0} e^{-\frac{E_{a}}{R\left(T^{*} T_{s}+T_{r}\right)}}\left(C_{A}^{*} C_{A, s}+C_{A, r}\right)
$$

In the above nonlinear term, it is difficult to separate the expression for reaction scale involving $\left(C_{A, s}, C_{A, r}, T_{s}, T_{r}\right)$ and the expression for dimensionless reaction term involving $\left(C_{A}^{*}, T^{*}\right)$. In the literature (Balaji et al., 2008; 
Krantz, 2007; Krantz et al., 2012), it is suggested that some characteristic maximum be used as the scale, but obtaining this characteristic maximum is not obvious and clear. In this paper, we address this issue of finding scales for such nonlinear terms.

Step 3: Once dimensionless variable definitions are introduced, dimensionless equations can be formed by dividing the equation by the dimensional coefficient of any particular term in the equation. For each of the above equations (Eq. (5) and Eq. (6)), one has three possibilities to form a dimensionless equation. For example, for Eq. (5)

$$
\begin{aligned}
\frac{d y_{1}^{*}}{d x^{*}} & =\frac{x_{s} f_{11, s}}{y_{1, s}} f_{11}^{*}+\frac{x_{s} f_{12, s}}{y_{1, s}} f_{12}^{*} \\
\frac{y_{1, s}}{x_{s} f_{11, s}} \frac{d y_{1}^{*}}{d x^{*}} & =f_{11}^{*}+\frac{f_{12, s}}{f_{11, s}} f_{12}^{*} \\
\frac{y_{1, s}}{x_{s} f_{12, s}} \frac{d y_{1}^{*}}{d x^{*}} & =\frac{f_{11, s}}{f_{12, s}} f_{11}^{*}+f_{12}^{*}
\end{aligned}
$$

In Eq. (10), Eq. (11) and Eq. (12), we have divided the dimensional coefficient of first, second and third terms, respectively. The dimensional coefficient has to be selected such that the resulting dimensionless equation is of order 1. This usually involves a trial and error method where each of these possibilities may need to be evaluated.

Step 4: After identifying particular form of dimensionless equation, then next step is to calculate scale and reference values involved in these equations. This is again done by a trial and error method by assuming that a particular dimensionless group is dominant in the equation and making it equal to 1. For example, by considering Eq. (10) and similar dimensionless equation for Eq. (6), scales for the dependent variables can be found by assuming dimensionless group in first term in these equations to be dominant. Scale for independent variable can be found from its maximum value and 
equating the corresponding dimensionless variable to 1.

$$
\frac{x_{s} f_{11, s}}{y_{1, s}}=1 ; \quad \frac{x_{s} f_{21, s}}{y_{2, s}}=1 ; \quad \frac{L}{x_{s}}=1
$$

The scales $y_{1, s}, y_{2, s}$ and $x_{s}$ can be found by solving Eq. (13) where $L$ is the maximum value for the independent variable.

Step 5: Reference value for a variable is found from the dimensionless initial condition by equating it to zero. Thus from Eq. (7), one can calculate $y_{1, r}$ and $y_{2, r}$ from,

$$
\begin{aligned}
& y_{1}^{*}\left(x^{*} x_{s}=0\right)=\frac{\left(y_{10}-y_{1, r}\right)}{y_{1, s}}=0 ; \Longrightarrow y_{1, r}=y_{10} \\
& y_{2}^{*}\left(x^{*} x_{s}=0\right)=\frac{\left(y_{20}-y_{2, r}\right)}{y_{2, s}}=0 ; \Longrightarrow y_{2, r}=y_{20}
\end{aligned}
$$

Once appropriate scale and reference values are found, they can be substituted in other dimensionless groups. If the resulting values of dimensionless groups are in the order of 1 or much less than 1 , then the calculated scales are retained. If the values are much greater than 1, then either the chosen dominant dimensionless group or the chosen form of dimensionless equation is incorrect and needs to be changed. As one can see, step 3 and step 4 involves trial and error. After identifying appropriate dimensionless equation, simplification of corresponding equations can be undertaken by examining the values of dimensionless groups. In the next section, we propose an improved version of scaling methodology described in the literature for efficient handling of nonlinear terms and for efficient calculation of scale and reference values.

\section{Proposed improvements in scaling analysis}

In the scaling analysis described in the literature, we see that handling of nonlinear terms during scaling analysis is not clear and not general. Also 
during scaling analysis, one need to follow a trial and error procedure during selection correct form of dimensionless equation and during calculation of scales. In this paper we propose an systematic approach where scaling analysis is general and intuitive to any given model equations. This new approach is explained through following steps

Step 1: Given a set of model equations with corresponding initial and boundary conditions, introduce the definition of dimensionless variable involving scale and reference values, for all dependent and independent variables. To make the procedure more general, introduce reference value for all the variables regardless for whether they start from zero or not.

Step 2: In addition to the variables, introduce dimensionless quantity definition involving only scale values, for all the terms which are formed from the linear or nonlinear combination of dependent and independent variables.

Step 3:Introduce these definitions into model equations and corresponding initial and boundary conditions and form dimensionless equations.

Step 4: Next step is to form algebraic equations for the calculation of scale and reference values. Following ideas help in forming such equations

1. In the dimensionless equation formed above, equate all the dimensionless variables and dimensionless terms to take the value of 1 . The reason for this equation is that we need value of all the dimensionless variables to vary in the order of one, hence we can safely assume these variables to take the value of 1 . This will result in algebraic equation involving only scale and reference values.

2. The dimensionless variables in inlet conditions (i.e. boundary conditions corresponding to initial value of independent variables) can be assumed to take the value of zero. This is to make all the dimensionless 
variables to start from zero.

3. The dimensionless variables in outlet condition (i.e. boundary conditions corresponding to final value of independent variables) can be assumed to take the value of 1 . This is to make all the dimensionless variables to take the value of 1 at the outlet condition. For initial value problems, one will not have this outlet condition, whereas this is present for boundary value problems.

4. For certain cases, because of the nature of the problem, actual variables might take a value of zero at outlet condition and in those cases, corresponding dimensionless variable can also be assumed to take a value of zero.

Step 5: Solving the resulting algebraic equations from above step gives appropriate scale and reference values which makes the dimensionless variables to vary in the order of 1.

Step 6: Once the scale and reference values are found, then it is easy to form appropriate dimensionless groups involving these values and asses their relative importance in the equation.

It is to be noted that the above scaling approach may not be applicable to model equations which exhibits steady state multiplicity. In such cases, one might be able to do scaling analysis corresponding to each steady state separately, but this needs to be explored. Also the proposed approach may not work for the case where equations exhibit multi regimes which could shift dynamically. One might have to work with parameter ranges at the time of preliminary scaling to more comprehensively address this issue and this needs further exploration. 


\subsection{Details of the proposed steps through an example}

Considering a set of equations, Eq. (1), Eq. (2) and Eq. (3), as given in the previous section, one need to introduce dimensionless variable definition for dependent variables $\left(y_{1}\right.$ and $\left.y_{2}\right)$, independent variable $x$ and dimensionless term definition for nonlinear (or linear) functions $\left(f_{11}, f_{12}, f_{21}, f_{22}\right)$. The definition of dimensionless variables for the independent and dependent variables are the same as in Eq. (4). The definition for the dimensionless terms is as follows

$$
f_{11}^{*}=\frac{f_{11}}{f_{11, s}} ; \quad f_{12}^{*}=\frac{f_{12}}{f_{12, s}} ; \quad f_{21}^{*}=\frac{f_{21}}{f_{21, s}} ; \quad f_{22}^{*}=\frac{f_{22}}{f_{22, s}}
$$

Introducing these definitions into model, results in following set of equations

$$
\begin{aligned}
\frac{d y_{1}^{*}}{d x^{*}} & =\frac{x_{s} f_{11, s}}{y_{1, s}} f_{11}^{*}+\frac{x_{s} f_{12, s}}{y_{1, s}} f_{12}^{*} \\
\frac{d y_{2}^{*}}{d x^{*}} & =\frac{x_{s} f_{21, s}}{y_{2, s}} f_{21}^{*}+\frac{x_{s} f_{22, s}}{y_{2, s}} f_{22}^{*} \\
f_{11}^{*} f_{11, s} & =f_{11}\left(y_{1}^{*} y_{1, s}+y_{1, r}, y_{2}^{*} y_{2, s}+y_{2, r}, x^{*} x_{s}+x_{r}\right) \\
f_{12}^{*} f_{12, s} & =f_{12}\left(y_{1}^{*} y_{1, s}+y_{1, r}, y_{2}^{*} y_{2, s}+y_{2, r}, x^{*} x_{s}+x_{r}\right) \\
f_{21}^{*} f_{21, s} & =f_{21}\left(y_{1}^{*} y_{1, s}+y_{1, r}, y_{2}^{*} y_{2, s}+y_{2, r}, x^{*} x_{s}+x_{r}\right) \\
f_{22}^{*} f_{22, s} & =f_{22}\left(y_{1}^{*} y_{1, s}+y_{1, r}, y_{2}^{*} y_{2, s}+y_{2, r}, x^{*} x_{s}+x_{r}\right)
\end{aligned}
$$

Introducing the definitions into inlet conditions

$$
\begin{aligned}
x^{*} x_{s}+x_{r} & =0 \\
y_{1}^{*} y_{1, s}+y_{1, r} & =y_{10} ; \quad y_{2}^{*} y_{2, s}+y_{2, r}=y_{20}
\end{aligned}
$$

Similarly at outlet condition, this becomes

$$
x^{*} x_{s}+x_{r}=L
$$


Now assume dimensionless variables and dimensionless terms in Eq. (17) to Eq. (22) to take the value of 1 . Similarly, assume the dimensionless variables in Eq. (23) and Eq. (24) to take the value of zero and the dimensionless variable in Eq. (25) to take the value of 1 . This results in following algebraic equations for the calculation of scale and reference values:

$$
\begin{aligned}
1 & =\frac{x_{s} f_{11, s}}{y_{1, s}}+\frac{x_{s} f_{12, s}}{y_{1, s}} \\
1 & =\frac{x_{s} f_{21, s}}{y_{2, s}}+\frac{x_{s} f_{22, s}}{y_{2, s}} \\
f_{11, s} & =f_{11}\left(y_{1, s}+y_{1, r}, y_{2, s}+y_{2, r}, x_{s}+x_{r}\right) \\
f_{12, s} & =f_{12}\left(y_{1, s}+y_{1, r}, y_{2, s}+y_{2, r}, x_{s}+x_{r}\right) \\
f_{21, s} & =f_{21}\left(y_{1, s}+y_{1, r}, y_{2, s}+y_{2, r}, x_{s}+x_{r}\right) \\
f_{22, s} & =f_{22}\left(y_{1, s}+y_{1, r}, y_{2, s}+y_{2, r}, x_{s}+x_{r}\right) \\
y_{1, r} & =y_{10} ; \quad y_{2, r}=y_{20} ; \\
x_{r} & =0 \\
x_{s}+x_{r} & =L
\end{aligned}
$$

Once scale and reference values are calculated by solving above algebraic equations, it is easy to form dimensionless groups, which can provide information about phenomena occurring at various scales. This information can be subsequently used in possible simplification of the equations.

\section{Examples of varying complexity to illustrate the proposed idea for scaling analysis}

In this section, we illustrate the proposed idea using various examples of different complexity. The first example consider model equations with single independent variable and the second example consider equations with 
two independent variables. In both the examples, we use Dirichlet type boundary conditions and in third example, we use Neumann type boundary condition. Finally, we consider an example involving DAEs with two independent variables and involving Neumann type boundary conditions. In all these examples, various steps explained in previous section are indicated at appropriate places.

\subsection{Example 1: Steady-state plug flow reactor}

The mathematical model of steady plug flow reactor is given by following differential equations

$$
\frac{d c_{A}}{d z}=-r_{A} ; \quad \frac{d T}{d z}=B_{1} r_{A} ; \quad r_{A}=k_{0} e^{\frac{-E_{a}}{R T}} C_{A}
$$

The boundary conditions corresponding to inlet are given by

$$
C_{A}(z=0)=C_{A 0} ; \quad T(z=0)=T_{0}
$$

The boundary condition corresponding to the outlet is given by $z=L$.

Step 1 \& 2: Define dimensionless quantities for the variables and terms involved in the equations

$$
z^{*} \equiv \frac{z-z_{r}}{z_{s}} ; \quad C_{A}^{*} \equiv \frac{C_{A}-C_{A, r}}{C_{A, s}} ; \quad T^{*} \equiv \frac{T-T_{r}}{T_{s}} ; \quad r_{A}^{*} \equiv \frac{r_{A}}{r_{A, s}}
$$

Step 3: Introducing the definitions into the model equations

$$
\begin{aligned}
\frac{C_{A, s}}{z_{s}}\left(\frac{d C_{A}}{d z}\right)^{*} & =-r_{A, s} r_{A}^{*} \\
\frac{T_{s}}{z_{s}}\left(\frac{d T}{d z}\right)^{*} & =B_{1} r_{A, s} r_{A}^{*} \\
r_{A, s} r_{A}^{*} & =k_{0} e^{-\frac{E_{a}}{R\left(T^{*} T_{s}+T_{r}\right)}}\left(C_{A}^{*} C_{A, s}+C_{A, r}\right)
\end{aligned}
$$


And introducing the definitions into the boundary conditions

$$
\begin{aligned}
C_{A}^{*} C_{A, s}\left(z^{*} z_{s}+z_{r}=0\right)+C_{A, r} & =C_{A, 0} \text { at inlet } \\
T^{*} T_{s}\left(z^{*} z_{s}+z_{r}=0\right)+T_{r} & =T_{0} \text { at inlet } \\
z^{*} z_{s}+z_{r} & =L \text { at outlet }
\end{aligned}
$$

Step 4:Assuming dimensionless variables taking the value of 1 in the model equations, we have the following algebraic equations

$$
\frac{c_{A, s}}{z_{s}}=-r_{A, s} ; \quad \frac{T_{s}}{z_{s}}=B_{1} r_{A, s} ; \quad r_{A, s}=k_{0} e^{\frac{-E_{a}}{R\left(T_{s}+T_{r}\right)}}\left(C_{A, s}+C_{A, r}\right)
$$

From the inlet conditions (assuming $C_{A}^{*}=T^{*}=z^{*}=0$ )

$$
C_{A, r}=C_{A, 0} ; \quad T_{r}=T_{0} ; \quad z_{r}=0
$$

From the outlet condition (assuming $z^{*}=1$ ) we have $z_{s}=L$.

The solution of the above algebraic equations provides appropriate scales and reference values that will make the corresponding dimensionless variable to vary in the order of 1.

\subsection{Example 2: Unsteady-state plug flow reactor}

In the unsteady plug flow reactor model, we have partial differential equations (PDEs) due to the presence of two independent variables, space and time. These PDEs can be converted to ordinary differential equations using method of lines and scale and reference values can be found as in previous example. In such case we will have as many scale and reference values for a variable as number of discretization points used in the method of lines procedure. Alternatively, we can also restrict the number of scale

and reference values to one for each variable and calculate the corresponding 
values. In this example, we illustrate a later case where we consider a single scale and reference value for each variable.

The mathematical model of unsteady plug flow reactor is given below

$$
\begin{aligned}
\frac{\partial C_{A}}{\partial t} & =-v_{1} \frac{\partial C_{A}}{\partial z}-r_{A} \\
\frac{\partial T}{\partial t} & =-v_{2} \frac{\partial T}{\partial z}+B_{2} r_{A} \\
r_{A} & =k_{0} e^{-\frac{E a}{R T}} C_{A}
\end{aligned}
$$

Boundary conditions corresponding to initial value of independent variables, i.e. inlet conditions and initial and conditions are given by

$$
C_{A}(z=0)=C_{A, 0} ; \quad T(z=0)=T_{0} ; \quad C_{A}(t=0)=f_{1} ; \quad T(t=0)=f_{2}
$$

Where $f_{1} \& f_{2}$ represent the initial profile of dependent variables at $t=0$. Boundary conditions corresponding to final value of independent variables are given by

$$
z=L ; \quad t=t_{\text {end }}
$$

Step 1 \& 2: Define dimensionless quantities for the variables and term involved in the equation

$$
z^{*} \equiv \frac{z-z_{r}}{z_{s}} ; \quad t^{*} \equiv \frac{t-t_{r}}{t_{s}} ; \quad C_{A}^{*} \equiv \frac{C_{A}-C_{A, r}}{C_{A, s}} ; \quad T^{*} \equiv \frac{T-T_{r}}{T_{s}} ; \quad r_{A}^{*} \equiv \frac{r_{A}}{r_{A, s}}
$$

Step 3: Introducing the definitions into the model equations

$$
\begin{aligned}
\frac{C_{A, s}}{t_{s}}\left(\frac{\partial C_{A}}{\partial t}\right)^{*} & =-v_{1} \frac{C_{A, s}}{L}\left(\frac{\partial C_{A}}{\partial z}\right)^{*}-r_{A, s} r_{A}^{*} \\
\frac{T_{s}}{t_{s}}\left(\frac{\partial T}{\partial t}\right)^{*} & =-v_{2} \frac{T_{s}}{L}\left(\frac{\partial T}{\partial z}\right)^{*}+B_{2} r_{A, s} r_{A}^{*} \\
r_{A, s} r_{A}^{*} & =k_{0} e^{-\frac{E_{a}}{R\left(T^{*} T_{s}+T_{r}\right)}}\left(C_{A}^{*} C_{A, s}+C_{A, r}\right)
\end{aligned}
$$


And introducing the definitions into the boundary conditions

$$
\begin{gathered}
C_{A}^{*} C_{A, s}\left(z^{*} z_{s}+z_{r}=0\right)+C_{A, r}=C_{A, 0} ; \quad T^{*} T_{s}\left(z^{*} z_{s}+z_{r}=0\right)+T_{r}=T_{0} \\
C_{A}^{*} C_{A, s}\left(t^{*} t_{s}+t_{r}=0\right)+C_{A, r}=f_{1} ; \quad T^{*} T_{s}\left(t^{*} t_{s}+t_{r}=0\right)+T_{r}=f_{2} \\
z^{*} z_{s}+z_{r}=L ; \quad t^{*} t_{s}+t_{r}=t_{\text {end }}
\end{gathered}
$$

Step 4: Assuming dimensionless variables take the value of 1 in the model equations, we have the following algebraic equations

$$
\begin{aligned}
\frac{C_{A, s}}{t_{s}} & =-v_{1} \frac{C_{A, s}}{z_{s}}-r_{A, s} \\
\frac{T_{s}}{t_{s}} & =-v_{2} \frac{T_{s}}{z_{s}}+B_{2} r_{A, s} \\
r_{A, s} & =k_{0} e^{\frac{-E_{a}}{R\left(T_{s}+T_{r}\right)}}\left(C_{A, s}+C_{A, r}\right)
\end{aligned}
$$

From the initial and inlet conditions assuming $C_{A}^{*}=T^{*}=z^{*}=t^{*}=0$

$$
C_{A, r}=C_{A, 0} ; \quad T_{r}=T_{0} ; \quad z_{r}=0 ; \quad t_{r}=0
$$

We can either assign the above values as the reference variables for $C_{A, r} \& T_{r}$ or utilize an average value from the initial conditions as given below,

$$
C_{A, r}=F_{1} ; \quad T_{r}=F_{2}
$$

$F_{1}$ and $F_{2}$ represent the average value obtained from the initial distribution of the dependent variables over the domain $(0, L)$. From the other boundary condition, by assuming $z^{*}=t^{*}=1$, we have

$$
z_{s}=L ; \quad t_{s}=t_{\text {end }}
$$

Solution of the above nonlinear algebraic equation will give appropriate scales and reference values. 
4.3. Example 3: Boundary value problem with Neumann-type boundary condition

In previous examples, reference values are found using boundary conditions and scales for dependent variables are found by solving algebraic equations. In this example we demonstrate how the reference values are found from the algebraic equations when Neumann-type boundary conditions are involved. The equations and the corresponding boundary conditions are given below

$$
\frac{d^{2} T}{d x^{2}}=f(T) ; \quad \frac{d T}{d x}(x=0)=y_{1} ; \quad \frac{d T}{d x}(x=L)=y_{2}
$$

Above equation can be rewritten as following two ODEs and corresponding boundary conditions

$$
\frac{d T}{d x}=a ; \quad \frac{d a}{d x}=f(T) ; \quad a(x=0)=y_{1} ; \quad a(x=L)=y_{2}
$$

Step 1,2 \& 3: Introducing dimensionless variable definitions corresponding to $T$ and $a$,

$$
\frac{T_{s}}{L}\left(\frac{d T}{d x}\right)^{*}=a^{*} a_{s}+a_{r} ; \quad \frac{a_{s}}{L}\left(\frac{d a}{d x}\right)^{*}=f\left(T^{*} T_{s}+T_{r}\right)
$$

Boundary conditions become

$$
a^{*} a_{s}\left(x^{*} x_{s}+x_{r}=0\right)+a_{r}=y_{1} ; \quad a^{*} a_{s}\left(x^{*} x_{s}+x_{r}=L\right)+a_{r}=y_{2}
$$

Step 4: Algebraic equations can be formed as before using the assumption of dimensionless variable taking the value of 1 or 0 :

$$
\frac{T_{s}}{L}=a_{s}+a_{r} ; \quad \frac{a_{s}}{L}=f\left(T_{s}+T_{r}\right)
$$

From the boundary conditions

$$
a_{r}=y_{1} ; \quad a_{s}+a_{r}=y_{2} ; \quad x_{r}=0 ; \quad x_{s}=L ;
$$

The solution to the above algebraic equations will provide both scale and reference values for the variable $T$ and $a$. 


\subsection{Example 4: Unsteady mathematical model of water gas shift reactor}

In this example, we consider an unsteady model of water gas shift (WGS) reactor which involves DAEs with 9 dependent variables, Neumann-type boundary condition and two equilibrium reactions with nonlinear kinetics. This example represent the case where actual value of one of the variables at outlet condition is zero and in such cases we make corresponding dimensionless variable also to take value of zero. For this example, numerical value of scales and reference values are calculated from the given values of parameter and boundary condition. This example will be extended later in this paper to show the possible simplifications that are possible from the assessment of resulting dimensionless groups. The unsteady model for WGS reactor and corresponding reaction kinetic equations are adopted from Mobed et al. (2014). This model contains hyperbolic type PDE for species balance and parabolic type PDE for energy balance. Later in this paper, we show that simplification of model equations based on dimensionless groups result in only hyperbolic type PDEs. Following two reactions are assumed to take place in the WGS reactor. Schematic of WGSR with corresponding boundary conditions is shown in Fig. 1

$$
\begin{aligned}
& \mathrm{CO}+\mathrm{H}_{2} \mathrm{O} \Longleftrightarrow \mathrm{CO}_{2}+\mathrm{H}_{2} \Longrightarrow \text { Water Gas shift reaction } \\
& \mathrm{COS}+\mathrm{H}_{2} \mathrm{O} \Longleftrightarrow \mathrm{CO}_{2}+\mathrm{H}_{2} \mathrm{~S} \Longrightarrow \text { Hydrolysis reaction }
\end{aligned}
$$

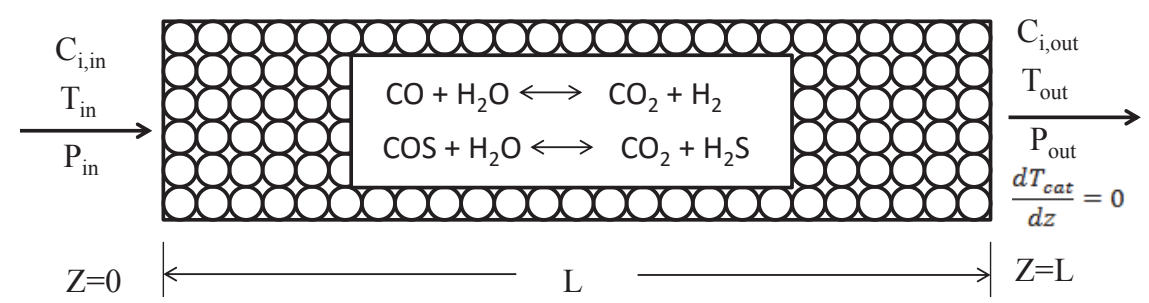

Figure 1: Schematic of WGSR with corresponding BCs 
Species balance $(i=1$ to 6$)$ :

$$
\begin{aligned}
& \frac{\partial C_{i}}{\partial t}=-\frac{G R T_{g}}{P} \frac{\partial C_{i}}{\partial z}-C_{i} \frac{G R T_{g}}{P}\left(\frac{1}{T_{g}} \frac{d T_{g}}{d z}-\frac{1}{P} \frac{d P}{d z}\right)+\left(r_{w g s} \gamma_{w g s, i}+r_{h y d} \gamma_{h y d, i}\right) \frac{1-\varepsilon}{\varepsilon} \\
& C_{1}=C_{C O} ; \quad C_{2}=C_{H_{2} O} ; \quad C_{3}=C_{C O_{2}} ; \quad C_{4}=C_{H_{2}} ; \quad C_{5}=C_{C O S} ; \\
& C_{6}=C_{H_{2} S}
\end{aligned}
$$

Pressure drop equation:

$$
\frac{d P}{d z}=-\frac{\rho_{g}}{d_{c a t}}\left(\frac{G R T_{g} \varepsilon}{P}\right)^{2}\left(\frac{1-\varepsilon}{\varepsilon^{3}}\right)\left(1.75+\frac{150 \mu P(1-\varepsilon)}{d_{c a t} \varepsilon G R T_{g} \rho_{g}}\right)
$$

Gas phase energy balance:

$$
\frac{\partial T_{g}}{\partial t}=\frac{1}{\rho_{g} C p_{g}}\left(-C p_{g} G \frac{\partial T_{g}}{\partial z}+\frac{h_{f} a_{c}}{\varepsilon}\left(T_{c a t}-T_{g}\right)\right)
$$

Catalyst phase energy balance:

$\frac{\partial T_{c a t}}{\partial t}=\frac{1}{\rho_{c a t} C p_{c a t}}\left(K_{c a t} \frac{\partial^{2} T_{c a t}}{\partial z^{2}}-\frac{h_{f} a_{c}}{1-\varepsilon}\left(T_{c a t}-T_{g}\right)+r_{w g s} \Delta H_{R, w g s}+r_{h y d} \Delta H_{R, h y d}\right)$

\section{Reaction kinetic equation:}

Water Gas Shift reaction:

$$
\begin{gathered}
r_{w g s}=-\Omega k_{w g s}\left(\frac{P}{101325}\right)^{\left(0.5-\frac{P}{50662500}\right)} \frac{R T_{\text {cat }}}{P}\left(C_{C O}-\frac{C_{C_{O} O_{2}} C_{H_{2}}}{K_{\text {eq,wgs }} C_{\mathrm{H}_{2} \mathrm{O}}}\right) \\
k_{\text {wgs }}=k_{01} e^{\frac{-E_{a_{1}}}{R T_{\text {cat }}}} ; \quad K_{\text {eq }, w g s}=e^{\left(\frac{4577.8}{T_{\text {cat }}}-4.33\right)}
\end{gathered}
$$

Hydrolysis reaction:

$$
\begin{gathered}
r_{h y d}=-k_{h y d}\left(\frac{10^{-3}\left(R T_{c a t} C_{C O S}\right)}{1+10^{-3}\left(K_{\text {eq }, h y d} R T_{c a t} C_{H_{2} O}\right)}\right) \\
k_{h y d}=k_{02} e^{\frac{-E_{a_{2}}}{R T_{\text {cat }}}} ; \quad K_{\text {eq,hyd }}=e^{\left(\frac{10010}{T_{\text {cat }}}-15.89\right)}
\end{gathered}
$$


Initial and boundary conditions:

$$
\begin{gathered}
z=0 ; \quad C_{i}=C_{i, i n} ; \quad T_{g}=T_{g, i n} ; \quad T_{\text {cat }}=T_{g, i n} ; \quad P=P_{\text {in }} \\
z=L ; \quad \frac{d T_{\text {cat }}}{d z}=0
\end{gathered}
$$

Equation for catalyst phase energy balance can be rewritten as following two differential equations

$$
\begin{aligned}
\frac{T_{c a t}}{z} & =T_{c a t, z} \\
\frac{\partial T_{c a t}}{\partial t} & =\frac{1}{\rho_{c a t} C p_{c a t}}\left(K_{c a t} \frac{\partial T_{c a t, z}}{\partial z}-\frac{h_{f} a_{c}}{1-\varepsilon}\left(T_{c a t}-T_{g}\right)+r_{w g s} \Delta H_{R, w g s}+r_{h y d} \Delta H_{R, h y d}\right)
\end{aligned}
$$

Boundary condition corresponding to new variable $T_{c a t, z}$ is given below

$$
z=L ; \quad T_{c a t, z}=0
$$

In the above model, in addition to reaction kinetic terms, one can find various terms involving combinations of dependent variables and these terms are listed below

$$
N_{1}=\frac{G R T_{g}}{P} ; \quad N_{2}=\frac{C_{i} G R}{P} ; \quad N_{3}=\frac{C_{i} G R T_{g}}{P^{2}} ; \quad N_{4}=\left(T_{c a t}-T_{g}\right)
$$

Step 1 \& 2: Dimensionless quantities are defined for the dependent and independent variables and for the terms involving these variables as given below

$$
\begin{gathered}
z^{*} \equiv \frac{z-z_{r}}{z_{s}} ; t^{*} \equiv \frac{t-t_{r}}{t_{s}} \\
C_{i}^{*} \equiv \frac{C_{i}-C_{i, r}}{C_{i, s}} ; \quad T_{g}^{*} \equiv \frac{T_{g}-T_{g, r}}{T_{g, s}} ; \quad T_{c a t}^{*} \equiv \frac{T_{c a t}-T_{c a t, r}}{T_{c a t, s}} ; \\
P^{*} \equiv \frac{P-P_{r}}{P_{s}} ; \quad T_{c a t, z}^{*} \equiv \frac{T_{c a t, z}-T_{c a t, z, r}}{T_{c a t, z, s}}
\end{gathered}
$$


$r_{w g s}^{*} \equiv \frac{r_{w g s}}{r_{w g s, s}} ; r_{h y d}^{*} \equiv \frac{r_{h y d}}{r_{h y d, s}} ; \quad N_{1} \equiv \frac{N_{1}}{N_{1, s}} ; \quad N_{2} \equiv \frac{N_{2}}{N_{2, s}} ; \quad N_{3} \equiv \frac{N_{3}}{N_{3, s}} ; N_{4} \equiv \frac{N_{4}}{N_{4, s}}$

Step 3 \& 4: Introducing the above definitions and assuming dimensionless variables to take the value of 1 following algebraic equations are formed

Algebraic equation for species concentration scale $(i=1$ to 6$)$ :

$\frac{C_{i, s}}{t_{s}}=-N_{1, s} \frac{C_{i, s}}{z_{s}}-N_{2, s} \frac{T_{g, s}}{z_{s}}+N_{3, s} \frac{P_{s}}{z_{s}}+\left(r_{w g s, s} \gamma_{w g s, i}+r_{h y d, s} \gamma_{h y d, i}\right) \frac{1-\varepsilon}{\varepsilon}$

$C_{1, s}=C_{C O, s} ; \quad C_{2, s}=C_{H_{2} O, s} ; \quad C_{3, s}=C_{C O_{2}, s} ; \quad C_{4, s}=C_{H_{2}, s} ; \quad C_{5, s}=$

$C_{C O S, s} ; \quad C_{6, s}=C_{H_{2} S, s}$

Algebraic equation for pressure scale:

$$
\frac{P_{s}}{z_{s}}=-\frac{1.75 \rho_{g}(1-\varepsilon) N_{1, s}^{2}}{d_{c a t} \varepsilon}-\frac{(1-\varepsilon)^{2}}{\varepsilon^{2}} \frac{150 \mu N_{1, s}}{d_{c a t}^{2}}
$$

Algebraic equation for gas phase temperature scale:

$$
\frac{T_{g, s}}{t_{s}}=-\frac{G}{\rho_{g}} \frac{T_{g, s}}{z_{s}}+\frac{h_{f} a_{c}}{\varepsilon \rho_{g} C p_{g}} N_{4, s}
$$

Algebraic equation for catalyst phase temperature scale:

$\frac{T_{c a t, s}}{t_{s}}=\frac{K_{c a t}}{\rho_{c a t} C p_{c a t}} \frac{T_{c a t, z, s}}{z_{s}}-\frac{h_{f} a_{c}}{\rho_{c a t} C p_{c a t}(1-\varepsilon)} N_{4, s}+\frac{\Delta H_{R, w g s}}{\rho_{c a t} C p_{c a t}} r_{w g s, s}+\frac{\Delta H_{R, h y d}}{\rho_{c a t} C p_{c a t}} r_{h y d, s}$

Algebraic equation for catalyst phase temperature gradient scale:

$$
\frac{T_{c a t, s}}{z_{s}}=\left(T_{c a t, z, s}-T_{c a t, z, r}\right)
$$

Introducing the definitions in to reaction kinetic terms and to terms involving combinations of dependent variables, following additional algebraic equations can be formed.

$$
r_{w g s, s}=-\Omega k_{w g s, s}\left(\frac{P_{s}+P_{r}}{101325}\right)^{\left(0.5-\frac{P_{s}+P_{r}}{50662500}\right)} \frac{R\left(T_{c a t, s}+T_{c a t, r}\right)}{P_{s}+P_{r}}
$$




$$
\begin{gathered}
\left(\left(C_{C O, s}+C_{C O, r}\right)-\frac{\left(C_{C O_{2}, s}+C_{C O_{2}, r}\right)\left(C_{H_{2}, s}+C_{H_{2}, r}\right)}{K_{e q, w g s, s}\left(C_{H_{2} O, s}+C_{H_{2} O, r}\right.}\right) \\
k_{w g s, s}=k_{01} e^{\frac{-E_{a}}{R\left(T_{c a t}, s+T_{c a t, r}\right)}} \\
r_{h y d, s}=-k_{h y d, s}\left(\frac{10^{-3} R\left(T_{c a t, s}+T_{c a t, r}\right)\left(C_{C O S, s}+C_{C O S, r}\right)}{1+10^{-3} K_{e q, h y d, s} R\left(T_{c a t, s}+T_{c a t, r}\right)\left(C_{H_{2} O, s}+C_{H_{2} O, r}\right.}\right) \\
\left(\frac{4577,8}{T_{c a t, s}+T_{c a t, r}}-4.33\right)
\end{gathered}
$$

From the inlet boundary conditions, we have

$$
\begin{gathered}
C_{i, r}=C_{i, i n} ; T_{g, r}=T_{g, i n} ; T_{c a t, r}=T_{g, i n} ; P_{r}=P_{i n} ; z_{r}=0 ; t_{r}=0 \\
z_{s}=L ; t_{s}=t_{\text {end }}
\end{gathered}
$$

As the catalyst phase temperature gradient variable takes the value of zero at outlet, we can make the corresponding dimensionless variable to take the value of zero at the outlet. This results in a reference value for catalyst temperature gradient to be zero, $T_{c a t, z, r}=0$. 


\section{Numerical calculation of scale and reference values for the given parameters for WGS reactor example}

In this section, scale and reference values are calculated using given parameters and boundary conditions for the WGS reactor model (step 5 in the proposed scaling analysis). The parameters and boundary condition values for WGS reactor model are given in Table 1 and these are introduced into the above algebraic equation(Eq. (88) - Eq. (100) and Eq. (102)) to calculate the appropriate values. The reference values are obtained from the corresponding boundary condition values, based on Eq. (101). The calculated scale values are given in Table 2. One can note that scales for the variables, decreasing along the length of the reactor (reactants concentration, pressure etc.) take negative values, so that corresponding dimensionless variables vary from 0 to near positive 1 . The calculation of scale and reference values does not involve forming 'appropriate dimensionless equation' and identifying 'appropriate dominant term' as followed in the literature and hence no trial and error procedure is involved.

\section{Verification of calculated scale and reference values using sim- ulation for WGS reactor example}

In this section, calculated scale and reference values are verified to make the corresponding dimensionless variables to vary in the order of 1 . This is to show that proposed method of scaling analysis results in scale and reference values that are appropriate in representing the model equation and hence can be used for further analysis such as model simplification. For this verification, first actual dimensional values of variables are obtained through simulation of WGS reactor model (Eq. (70) - Eq. (79)). Then 
Table 1: Parameters and inlet conditions for the WGS reactor

\begin{tabular}{|c|c|c|}
\hline Parameter & Value & \\
\hline Length of the reactor, $(\mathrm{L}, m)$ & 29 & \\
\hline Porosity of bed, $\varepsilon$ & 0.38 & \\
\hline Heat transfer coefficient, $\left(h_{f}, \frac{W}{m^{2} K}\right)$ & 100 & \\
\hline Heat of reaction for WGS, $\left(\Delta H_{w g s}, \frac{J}{m o l}\right)$ & $3.85 \times 10^{4}$ & \\
\hline Heat of reaction for Hydrolysis, $\left(\Delta H_{h y d}, \frac{J}{m o l}\right)$ & $3.1 \times 10^{4}$ & \\
\hline Average density of gas, $\left(\rho_{g}, \frac{m o l}{m^{3}}\right)$ & 20.5 & \\
\hline Viscosity of gas mixture, $\left(\mu_{g}, \frac{N-s}{m^{2}}\right)$ & $0.25 \times 10^{-4}$ & \\
\hline Specific heat of gas, $\left(C p_{g}, \frac{J}{m o l-K}\right)$ & 35 & \\
\hline Density of catalyst, $\left(\rho_{\text {cat }}, \frac{\mathrm{kg}}{\mathrm{m}^{3}}\right)$ & 650 & \\
\hline Specific heat of catalyst, $\left(C p_{c a t}, \frac{\mathrm{J}}{\mathrm{kg}-K}\right)$ & 880 & \\
\hline Specific area per unit volume of catalyst, $\left(a_{c}, \frac{m^{2}}{m^{3}}\right)$ & $1.69 \times 10^{3}$ & \\
\hline Conductivity of catalyst, $\left(K, \frac{W}{m-K}\right)$ & 35 & \\
\hline Diameter of catalyst, $\left(d_{c a t}, m\right)$ & 0.0035 & \\
\hline Inlet condition & $\begin{array}{l}\text { Values before } \\
\text { step change }\end{array}$ & $\begin{array}{l}\text { Values after } \\
\text { step change }\end{array}$ \\
\hline$C_{C O, i n},\left(\frac{m o l}{m^{3}}\right)$ & 246.34 & 238.64 \\
\hline$C_{H_{2} O, i n},\left(\frac{m o l}{m^{3}}\right)$ & 488.97 & 473.69 \\
\hline$C_{C O_{2}, i n},\left(\frac{m o l}{m^{3}}\right)$ & 95.02 & 92.05 \\
\hline$C_{H_{2}, i n},\left(\frac{m o l}{m^{3}}\right)$ & 234.65 & 227.32 \\
\hline$C_{C O S, i n},\left(\frac{m o l}{m^{3}}\right)$ & 0.0963 & 0.0933 \\
\hline$C_{H_{2} S, i n},\left(\frac{m o l}{m^{3}}\right)$ & 4.98 & 4.82 \\
\hline$T_{i n},(K)$ & 620 & 640 \\
\hline$P_{\text {in }},(P a)$ & $5.52 \times 10^{6}$ & $5.52 \times 10^{6}$ \\
\hline
\end{tabular}


Table 2: Values of scales for the variables in the WGS reactor

\begin{tabular}{llll}
\hline Scales & Value & Scales & Value \\
\hline$C_{C O, s}$ & -87.487 & $N_{2, C O, s}$ & 0.149 \\
$C_{H_{2} O, s}$ & -119.558 & $N_{2, H_{2} O, s}$ & 0.350 \\
$C_{C O_{2}, s}$ & 42.556 & $N_{2, C O_{2}, s}$ & 0.133 \\
$C_{H_{2}, s}$ & 24.066 & $N_{2, H_{2}, s}$ & 0.249 \\
$C_{C O S, s}$ & -0.0913 & $N_{2, C O S, s}$ & $1.9744 \times 10^{-6}$ \\
$C_{H_{2} S, s}$ & -0.578 & $N_{2, H_{2} S, s}$ & 0.0042 \\
$T_{g, s}$ & 55.358 & $N_{3, C O, s}$ & $2.035 \times 10^{-5}$ \\
$T_{c a t, s}$ & 55.449 & $N_{3, H_{2} O, s}$ & $4.769 \times 10^{-5}$ \\
$P_{s}$ & $-4.1 \times 10^{5}$ & $N_{3, C O_{2}, s}$ & $1.813 \times 10^{-5}$ \\
$z_{s}$ & 29 & $N_{3, H_{2}, s}$ & $3.385 \times 10^{-5}$ \\
$t_{s}$ & 2800 & $N_{3, C O S, s}$ & $2.689 \times 10^{-10}$ \\
$r_{w g s, s}$ & -0.939 & $N_{3, H_{2} S, s}$ & $5.713 \times 10^{-7}$ \\
$r_{h y d, s}$ & -0.0013 & $N_{1, s}$ & 0.688 \\
& & $N_{4, s}$ & 0.0912 \\
\hline
\end{tabular}


these actual values and calculated scale and reference values are substituted into Eq. (84) to Eq. (87) to obtain values for dimensionless variables.

Actual values are obtained from the simulation of WGS reactor model by introducing a step change in the steady-state inlet values. In Table 1, values for the inlet conditions before the step change (steady state inlet values) and after the step change (values for unsteady simulation) are given. Unsteady simulation is carried out from an initial time value of 0 second to 2800 second. The simulated results are plotted in four figures (Fig. 2 Fig. 5) for one of the variables $C O$. In first two figures, steady state results are plotted for the dimensional $C O$ concentration and for the corresponding dimensionless variable. In the next two figures, unsteady simulation results are plotted, using the value of the variable at the exit of the reactor, again for the dimensional $C O$ concentration and for the corresponding dimensionless variable. From the plots (Fig. 3 and Fig. 5), it can be observed that all the dimensionless variables vary between near zero to near 1 . This means the calculated scale and reference values are good enough in representing the magnitude of variation of the actual variables. Hence these values can be used for further analysis such as model simplification, developing analytical solutions to different regions of interest, etc.

From the simulated results one can also observe that for steady state value (Fig. 3), the dimensionless variable $\left(C O^{*}\right)$ vary from exactly zero to near 1, whereas for the unsteady values (Fig. 5), $C O^{*}$ vary from near zero to near 1 . This is because for steady state, inlet values are taken to be the reference values and this makes the steady state dimensionless variables to take the value of zero at inlet. Whereas for unsteady state, we have two choices for the calculation of reference and scale values as indicated in the illustrative example 2 , of section 4.2 . One choice uses the 


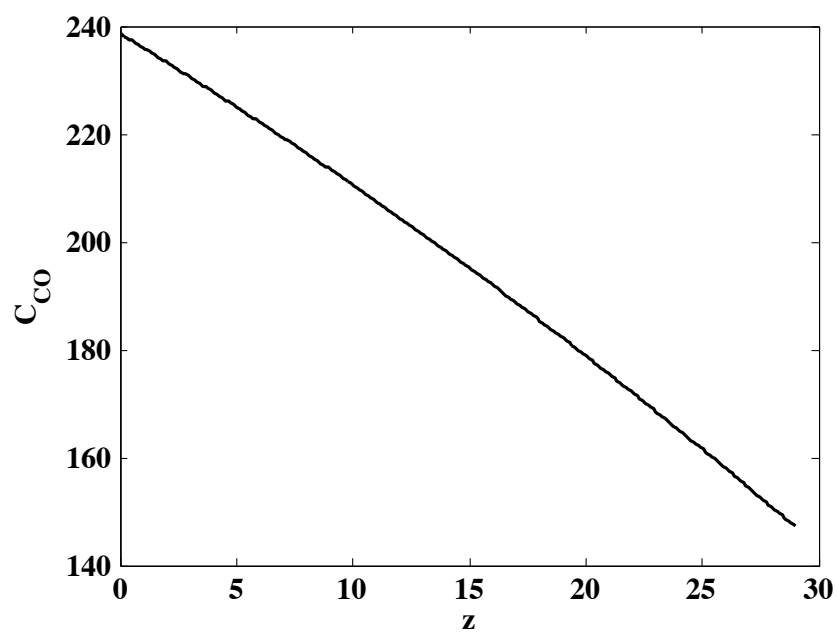

Figure 2: Simulated dimensional steady state result for $\mathrm{CO}$ concentration $\left(\mathrm{mol} / \mathrm{m}^{3}\right)$

initial conditions and other choice uses inlet condition. In the former case, there are many scale and reference values for each variable, corresponding to number of discretization points and in the later case we have single scale and reference value. As we have used only the inlet values as reference values, dimensionless variables at the discretization points other than the inlet do not take the exact value of zero at dimensionless time equal to zero. Nevertheless we see that values of dimensionless variables are in the order of 1 and this means that the calculated scale and reference values form a representative of the unsteady variations of WGS reactor model. Hence, any simplifications of model equations using these values will be reasonable and closely represent the behavior of corresponding detailed model. 


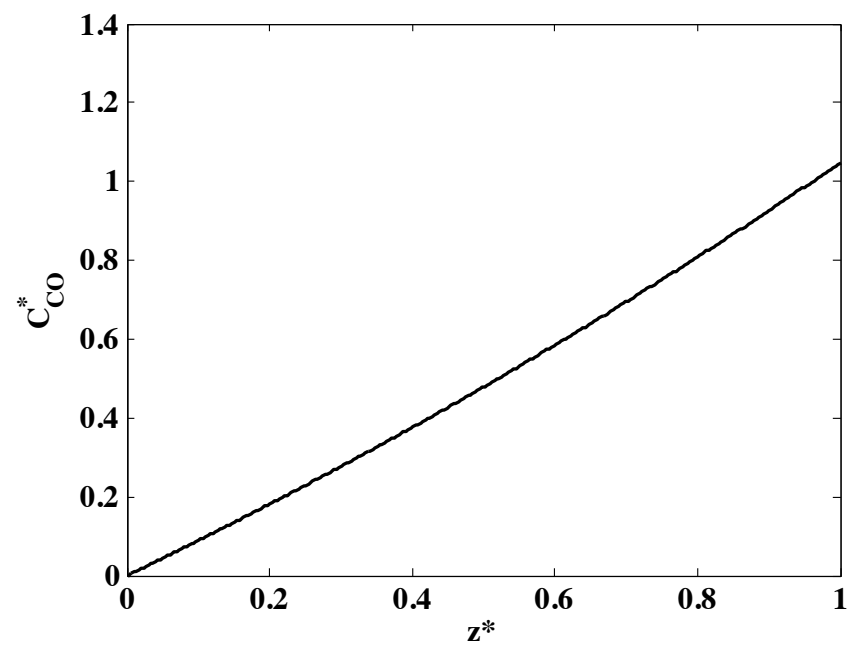

Figure 3: Calculated dimensionless steady state result for $\mathrm{CO}$ concentration using scale and reference values

\section{Simplification of the model based on the dimensionless groups - WGS reactor example}

This section focuses on application of scaling analysis in simplifying the model equations. We analyze the model equations of WGS reactor, using dimensionless groups formed from the calculated scale and reference values. Each term in a model equation represent some phenomena and ratio of two such phenomenon form a dimensionless group. Once scale and reference values are calculated, value of these phenomenon can easily be calculated and from which dominant phenomenon (phenomenon with higher value) can easily be identified. This dominant phenomenon can be divided throughout the equation to form dimensionless equation involving dimensionless groups. Thus formation of dimensionless equation does not involve any trial and error procedure. In the resulting dimensionless equation, whenever the value of a dimensionless group is much less than 1, then the phenomenon present in 


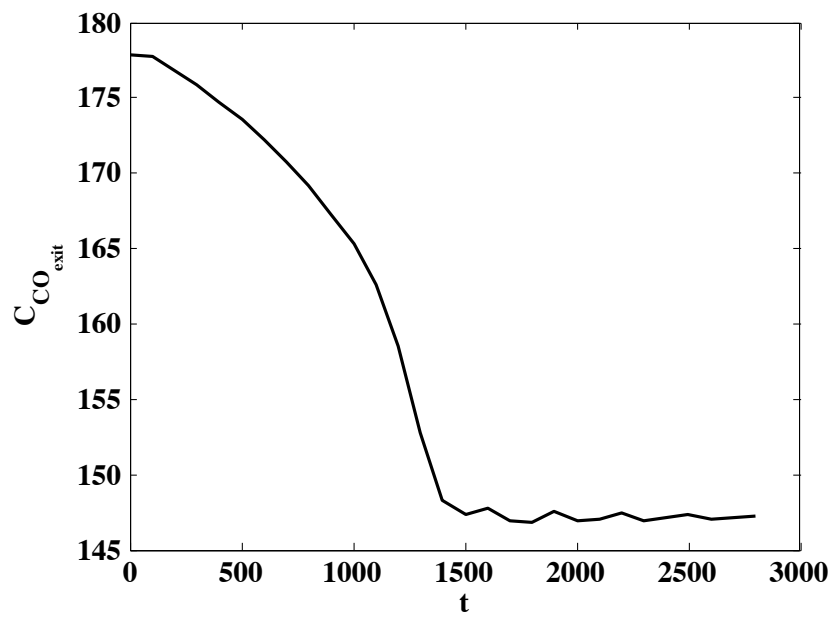

Figure 4: Simulated dimensional unsteady state result for CO concentration $\left(\mathrm{mol} / \mathrm{m}^{3}\right)$

numerator of that group can be eliminated while simplifying the model. In the following paragraph, we list all the phenomena and the corresponding terms representing these phenomena present in the model equations of WGS reactor.

In the unsteady species balance for the WGS reactor model, we note the following phenomena:

- Rate of accumulation of species per unit volume and is represented by, $\frac{C_{i, s}}{t_{s}}$

- Rate of convection of species per unit volume due to concentration variation and is represented by,$N_{1, s} \frac{C_{i, s}}{z_{s}}$

- Rate of convection of species per unit volume due to temperature variation and is represented by, $N_{2, s} \frac{T_{g, s}}{z_{s}}$

- Rate of convection of species per unit volume due to pressure variation and is represented by, $N_{3, s} \frac{P_{s}}{z_{s}}$ 


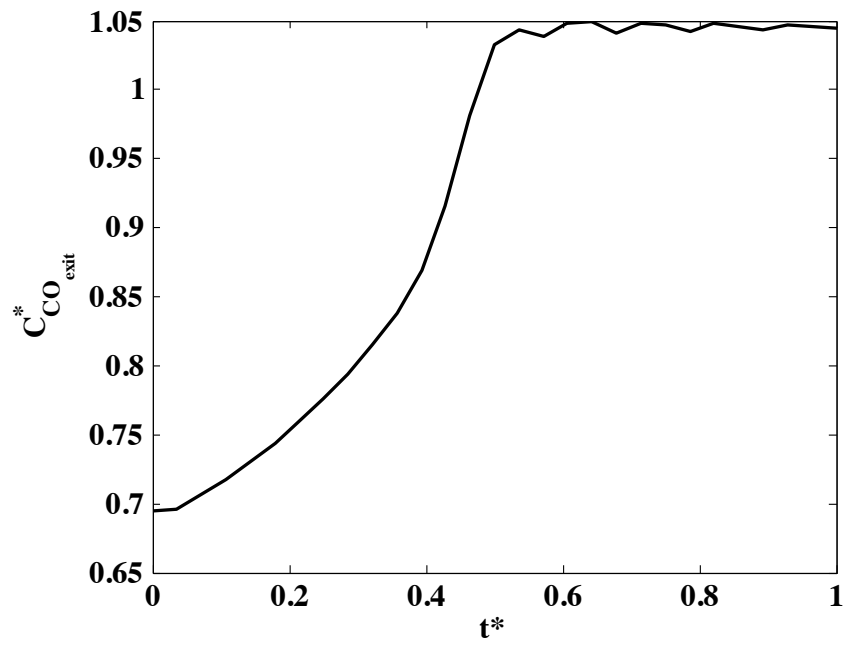

Figure 5: Calculated dimensionless unsteady state result for CO concentration using scale and reference values

- Rate of production/depletion of species per unit volume due to water gas shift reaction and is represented by, $r_{w g s, s} \gamma_{i}\left(\frac{1-\varepsilon}{\varepsilon}\right)$

- Rate of production/depletion of species per unit volume due to hydrolysis reaction and is represented by, $r_{h y d, s} \gamma_{i}\left(\frac{1-\varepsilon}{\varepsilon}\right)$

The value for a phenomenon can be found from the scale and reference values. It is observed that value for convection of species due to concentration gradient is high compared to other phenomenon in the species balance equations. Hence, the convection phenomenon is chosen to form dimensionless groups. In Table 3, we list the magnitude of dimensionless groups formed from the species balance equations. One can note that dimensionless group 5 \& 6 in Table 3 represent Damkohler number and similarly one can identify other named dimensionless groups.

Based on the values in Table 3, one can make various conclusions. From the value of dimensionless group 6, one can conclude that contribution of 
Table 3: Values of dimensionless groups for species balance equation of WGS reactor model

\begin{tabular}{|c|c|c|c|c|c|c|}
\hline $\begin{array}{l}\text { Species } \\
\text { balance }\end{array}$ & $\begin{array}{l}\text { Group } 1 \\
\frac{z_{s}}{N_{1, s} t_{s}}\end{array}$ & Group 2 & $\begin{array}{l}\text { Group } 3 \\
\frac{N_{2, s} T_{g, s}}{N_{1, s} C_{i, s}}\end{array}$ & $\begin{array}{l}\text { Group } 4 \\
\frac{N_{3, s} P_{s}}{N_{1, s} C_{i, s}}\end{array}$ & $\begin{array}{l}\text { Group } 5 \\
\frac{r_{w g s, s} \gamma_{i} z_{s}(1-\varepsilon)}{\varepsilon N_{1, s} C_{i, s}}\end{array}$ & $\begin{array}{l}\text { Group } 6 \\
\frac{r_{h y d, s} \gamma_{i} z_{s}(1-\varepsilon)}{\varepsilon N_{1, s} C_{i, s}}\end{array}$ \\
\hline$C_{C O}$ & 0.0150 & 1 & 0.137 & 0.1387 & 0.453 & 0 \\
\hline $\mathrm{C}_{\mathrm{H}_{2} \mathrm{O}}$ & 0.0153 & 1 & 0.24 & 0.243 & 0.55 & $7.62 \times 10^{-4}$ \\
\hline$C_{C_{2}}$ & 0.0150 & 1 & 0.252 & 0.253 & 1.52 & $2.1 \times 10^{-3}$ \\
\hline$C_{H_{2}}$ & 0.0154 & 1 & 0.85 & 0.855 & 2.74 & 0 \\
\hline$C_{C O S}$ & 0.0151 & 1 & 0.174 & $1.76 \times 10^{-3}$ & 0 & 0.98 \\
\hline$C_{H_{2} S}$ & 0.0150 & 1 & 0.585 & 0.59 & 0 & 0.155 \\
\hline
\end{tabular}

species concentration $\mathrm{C}_{\mathrm{H}_{2} \mathrm{O}}$ and $\mathrm{C}_{\mathrm{CO}_{2}}$ from hydrolysis reaction is low and hence the term involving this reaction for this species balance can be neglected. Similarly from dimensionless group 4, it is observed that the convection of species concentration $C_{C O S}$ due to pressure gradient is low and this term can be neglected. Further, from the value of dimensionless group 1 , one can observe that for all the species balance, rate of accumulation is smaller than convection and this implies unsteady behavior of the species balance is significant only for a short duration. This duration can be found by equating this dimensionless group to 1 and calculating the resulting time as follows

$$
\frac{z_{s}}{N_{1, s} \delta_{c_{i} t, s}}=1 \Rightarrow \delta_{c_{i} t, s}=42 \text { seconds }
$$

The shorter time taken by the species balance to reach the steady-state can also be verified through simulation of WGS reactor under isothermal and 
isobaric condition, i.e. simulating only the species balance equations. From Fig. 6 one can observe that $C O$ concentration reach steady-state at around 50 seconds and similar trend is also observed for other species.

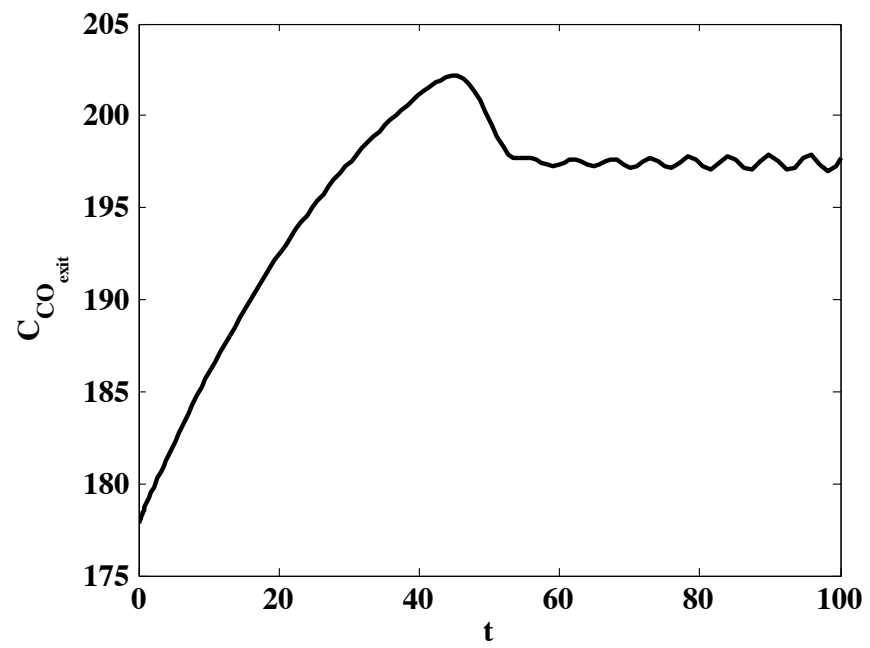

Figure 6: Unsteady behavior of species balance equation under isothermal and isobaric condition - dimensional $C O$ concentration $\left(\mathrm{mol} / \mathrm{m}^{3}\right)$

In the unsteady-state thermal balance for the gas phase, we note the following phenomena:

- Rate of accumulation of gas phase energy per unit volume and is represented by, $\frac{\rho_{g} C p_{g} T_{g, s}}{t_{s}}$

- Rate of convection of gas phase energy per unit volume and is represented by, $\frac{G C p_{g} T_{g, s}}{z_{s}}$

- Rate of transfer of gas phase energy per unit volume and is represented by, $\frac{h_{f} a_{c} N_{4, s}}{\varepsilon}$

Similarly, in the unsteady-state thermal balance for the catalyst phase, we have following phenomena 
- Rate of accumulation of catalyst phase energy per unit volume and is represented by, $\frac{\rho_{c a t} C p_{c a t} T_{c a t, s}}{t_{s}}$

- Rate of conduction of catalyst phase energy per unit volume and is represented by, $\frac{K_{c a t} T_{c a t, s}}{z_{s}^{2}}$

- Rate of transfer of catalyst phase energy per unit volume and is represented by, $\frac{h_{f} a_{c} N_{4, s}}{1-\varepsilon}$

- Rate of production of catalyst phase energy per unit volume due to water gas shift reaction and is represented by, $r_{w g s, s} \Delta H_{R, w g s}$

- Rate of production of catalyst phase energy per unit volume due to hydrolysis reaction and is represented by, $r_{h y d, s} \Delta H_{R, h y d}$

In Table 4, we list the magnitude of dimensionless groups formed by dividing each phenomenon in the gas phase energy balance with the convective flux phenomenon and similarly by dividing each phenomenon in the catalyst phase energy balance with the phenomenon that represents energy production from the water gas shift reaction. From the dimensionless group values in Table 4, we can observe that rate of accumulation for gas phase energy is negligible compared to the convective flux of energy and this means unsteady behavior of gas phase energy is significant only for a short duration. This is given by:

$$
\frac{\rho_{g} z_{s}}{G \delta_{T t, s}}=1 \Rightarrow \delta_{T_{R} t, s} \approx 1 \text { seconds }
$$

Similarly, rate of energy conduction through catalyst phase is negligible compared to rate of heat generation from water gas shift reaction and also rate of heat generation from hydrolysis reaction is negligible. Further, from the comparison of scale and reference values of gas phase and catalyst phase 
Table 4: Values of dimensionless groups for energy balance equation of WGS reactor model

\begin{tabular}{llllll}
\hline $\begin{array}{l}\text { Energy } \\
\text { balance }\end{array}$ & $\begin{array}{l}\text { Group 1 } \\
\frac{\rho_{g} z_{s}}{G t_{s}}\end{array}$ & Group 2 & $\begin{array}{l}\text { Group 3 } \\
h_{f} a_{c} z_{s} N_{4, s}\end{array}$ & Group 4 & Group 5 \\
\hline$T_{g} \varepsilon p_{g} T_{g, s}$ & & \\
\hline Energy & $3.5 \times 10^{-4}$ & 1 & 0.99 & - & - \\
\hline balance & $\frac{\rho_{\text {cat }} C p_{\text {cat }} T_{\text {cat }, s}}{r_{w g s, s} \Delta H_{R, w g s} t_{s}}$ & $\frac{K_{\text {cat }} T_{\text {cat }, s}}{r_{w g s, s} \Delta H_{R, w g s} z_{s}^{2}}$ & $\frac{h_{f} a_{c} N_{4, s}}{(1-\varepsilon) r_{w g s, s} \Delta H_{R, w g s}}$ & Group 4 & Group 5 \\
\hline$T_{\text {cat }}$ & 0.313 & $6.39 \times 10^{-5}$ & 0.69 & 1 & $\frac{r_{h y d, s} \Delta H_{R, h y d}}{r_{w g s, s} \Delta H_{R, w g s}}$ \\
\hline
\end{tabular}

Table 5: Values of dimensionless groups for new energy balance equation

\begin{tabular}{llll}
\hline $\begin{array}{l}\text { Energy } \\
\text { balance }\end{array}$ & $\begin{array}{l}\text { Group 1 } \\
\frac{\rho_{\text {cat }} C p_{\text {cat }}(1-\varepsilon) z_{s}}{G \varepsilon C p_{g} T_{s}}\end{array}$ & Group 2 & $\begin{array}{l}\text { Group 3 } \\
(1-\varepsilon) r_{w g s, s} \Delta H_{R, w g s} z_{s}\end{array}$ \\
\hline$T$ & 0.455 & 1 & $1.45 C p_{g} T_{g, s}$ \\
\hline
\end{tabular}

temperature, one can observe that the values are close, this means that both the temperatures vary in a similar manner along the length of the reactor. Hence, both the temperatures can be considered to be equal. Considering all the simplifications mentioned for energy balance, a new energy balance equation involving only single temperature variable can be written as:

$$
\frac{\partial T}{\partial t}=\frac{1}{\rho_{c a t} C p_{c a t}}\left(\frac{-G \varepsilon C p_{g}}{1-\varepsilon} \frac{\partial T}{\partial z}+r_{w g s} \Delta H_{R, w g s}\right)
$$

Based on the new energy balance, new dimensionless groups can be formed as shown below in the Table 5. From the dimensionless group 1 in Table 5, one can find the duration of unsteady behavior of new energy balance as 
Table 6: Values of dimensionless groups for pressure drop equation

\begin{tabular}{llll}
\hline $\begin{array}{l}\text { Pressure } \\
\text { drop }\end{array}$ & Group 1 & $\begin{array}{l}\text { Group 2 } \\
1.75 \rho_{g}(1-\varepsilon) N_{1, s}^{2} z_{s}\end{array}$ & $\begin{array}{l}\text { Group 3 } \\
P_{s} d_{c a t} \varepsilon\end{array}$ \\
\hline$P$ & 1 & 0.55 & 0.03 \\
\hline
\end{tabular}

given below

$$
\frac{\rho_{c a t} C p_{c a t}(1-\varepsilon) z_{s}}{G \varepsilon C p_{g} \delta_{T t, s}}=1 \Rightarrow \delta_{T t, s} \approx 1274 \text { seconds }
$$

Comparing the unsteady state duration for species balance (around 50 seconds) and for energy balance (around 1274 seconds), we see that dynamics of species balance is mostly governed by the dynamics of energy balance. This means we can easily assume the species balance to be at quasi steady-state during the simulation of WGS reactor model. Similar analysis can be done for pressure drop equation and the value of dimensionless groups in Table 6 indicates that all the terms in this equation are important and no further simplification is possible.

Considering all the simplifications mentioned above, the following simplified model can be derived.

Simplified Species balance:

$$
\begin{gathered}
\frac{d C_{i}}{d z}=-C_{i}\left(\frac{1}{T_{g}} \frac{d T_{g}}{d z}-\frac{1}{P} \frac{d P}{d z}\right)+\left(r_{w g s} \gamma_{w g s, i}\right) \frac{(1-\varepsilon) P}{G R T_{g} \varepsilon} \\
C_{1}=C_{C O} ; \quad C_{2}=C_{H_{2} O} ; \quad C_{3}=C_{C O_{2}} ; \quad C_{4}=C_{H_{2}} \\
\frac{d C_{\cos }}{d z}=-C_{\cos }\left(\frac{1}{T_{g}} \frac{d T_{g}}{d z}\right)+\left(r_{h y d} \gamma_{h y d, c o s}\right) \frac{(1-\varepsilon) P}{G R T_{g} \varepsilon} \\
\frac{d C_{H_{2} S}}{d z}=-C_{H_{2} S}\left(\frac{1}{T_{g}} \frac{d T_{g}}{d z}-\frac{1}{P} \frac{d P}{d z}\right)+\left(r_{h y d} \gamma_{h y d, H_{2} S}\right) \frac{(1-\varepsilon) P}{G R T_{g} \varepsilon}
\end{gathered}
$$




\section{Pressure drop equation:}

$$
\frac{d P}{d z}=-\frac{\rho_{g}}{d_{c a t}}\left(\frac{G R T_{g} \varepsilon}{P}\right)^{2}\left(\frac{1-\varepsilon}{\varepsilon^{3}}\right)\left(1.75+\frac{150 \mu P(1-\varepsilon)}{d_{c a t} \varepsilon G R T_{g} \rho_{g}}\right)
$$

\section{Simplified energy balance:}

$$
\frac{\partial T}{\partial t}=\frac{1}{\rho_{c a t} C p_{c a t}}\left(-\frac{G \varepsilon C p_{g}}{1-\varepsilon} \frac{\partial T}{\partial z}+r_{w g s} \Delta H_{R, w g s}\right)
$$

The inlet conditions and reaction kinetic equations remain same as in detailed model. The simplifications from scaling analysis results in conversion of parabolic type model to hyperbolic type PDE model. Further, energy balance equation for gas phase and catalyst phase is simplified to a single energy balance and this resulted in removing one boundary condition.

\section{Simulation and comparison of the detailed and simplified mod- els}

The simplified model obtained through scaling analysis is tested by comparing the simulation results with that generated from the detailed model. The percentage change in the average values of the variables between detailed and simplified model and the computational load are considered as metrics for comparison. These metrics represents the accuracy of the simplified model and its computational efficiency compared to the corresponding detailed model. For example, the average difference in the temperature during unsteady simulation between two models is given by

$T_{\text {avg }, \text { error }}=\frac{\left|\frac{1}{t_{\text {end }} L} \int_{0}^{t_{\text {end }}} \int_{0}^{L} T_{\text {detailed }} d z d t-\frac{1}{t_{\text {end }} L} \int_{0}^{t_{\text {end }}} \int_{0}^{L} T_{\text {simplified }} d z d t\right|}{\frac{1}{t_{\text {end }} L} \int_{0}^{t_{\text {end }}} \int_{0}^{L} T_{\text {detailed }} d z d t} \times 100$

In the above equation, the integral is calculated numerically based on the values of the corresponding variables. Computational load is measured in 
Table 7: Comparison of the reduced model vs. the detailed model

\begin{tabular}{lll}
\hline Variable/Model & Measure & Value \\
\hline$C_{c o}$ & $C_{c o, a v g . e r r o r}$ & 0.0057 \\
$C_{H_{2} O}$ & $C_{H_{2} \text { O,avg.error }}$ & 0.0089 \\
$C_{c 0_{2}}$ & $C_{c o_{2}, \text { avg.error }}$ & 0.0953 \\
$C_{H_{2}}$ & $C_{H_{2}, \text { avg.error }}$ & 0.0189 \\
$C_{c o s}$ & $C_{\text {cos,avg.error }}$ & 0.7031 \\
$C_{H_{2} S}$ & $C_{H_{2} \text { S,avg.error }}$ & 0.004 \\
$T_{g}$ & $T_{g, \text { avg.error }}$ & 0.0154 \\
$T_{c a t}$ & $T_{\text {cat,avg.error }}$ & 0.0276 \\
$P$ & $P_{\text {avg.error }}$ & $7.52 \times 10^{-4}$ \\
Detailed Model & Computational Load,s & 144.2 \\
Simplified Model & Computational Load,s & 98.9 \\
\hline
\end{tabular}

terms of time taken for unsteady simulation. Table. 7 shows the values for these metrics and the values shows that error percentage is quite small that one can consider the simplified model as a good approximation that can be used in lieu of the detailed model for the given parameter values and inlet conditions. The computational load is lower and is about only $68 \%$ of the computational load of the detailed model. Fig. 7 and Fig. 8 compares steady state and unsteady state simulation results for $C O$ between detailed and simplified model. The scaling analysis results in conversion of a parabolic PDE model to first order hyperbolic PDE model. The parabolic PDE nature comes from the conduction term in the original energy balance equation. The scaling analysis reveals that conduction term is insignificant 
and also both gas phase and catalyst phase temperatures can be considered to be varying similarly. This results in the removal of one of the equations for the energy balance and reveals the true nature of PDE, which is first order hyperbolic. This is an important observation, because further simplification of the model through order reduction depends on the type of PDE that is being solved. For parabolic type PDEs, proper orthogonal decomposition is normally used for the order reduction (for example, Shvartsman et al. (2000)), whereas for the hyperbolic PDEs, method of characteristic is used as the order reduction method (Munusamy et al., 2013, 2014). Hence the obtained model can be further simplified through order reduction using method of characteristics.

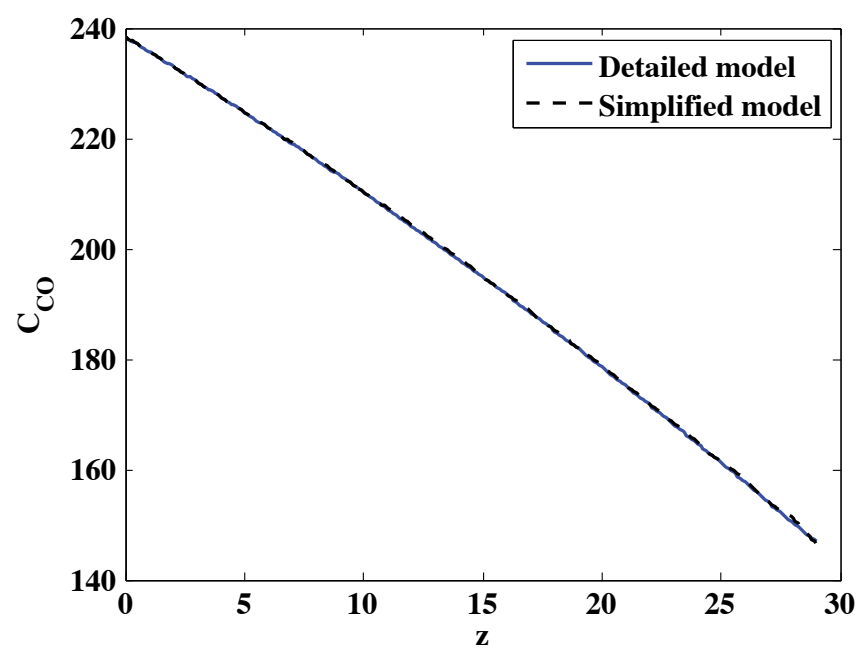

Figure 7: Comparison of steady state results between detailed and simplified model for $\mathrm{CO}$ concentration $\left(\mathrm{mol} / \mathrm{m}^{3}\right)$ 


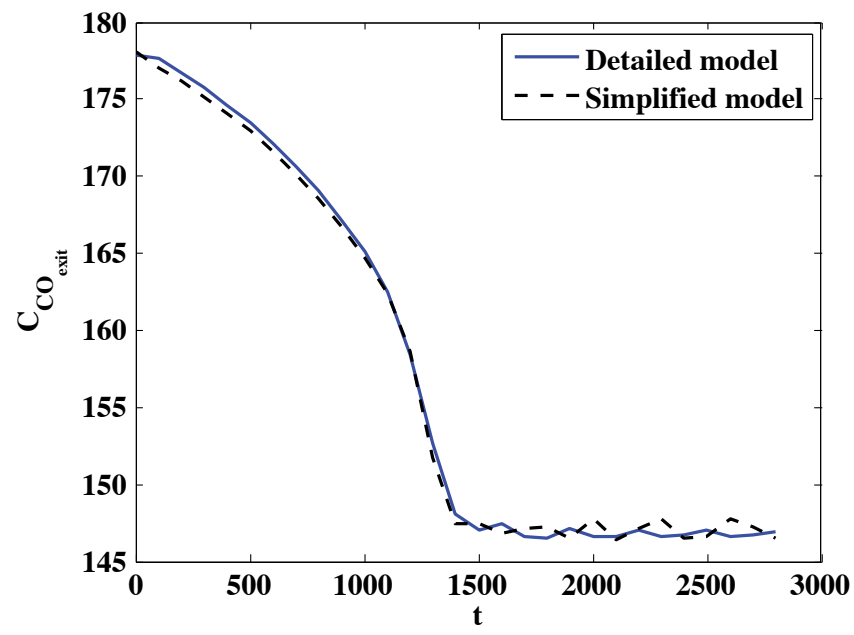

Figure 8: Comparison of unsteady state results between detailed and simplified model for $\mathrm{CO}$ concentration $\left(\mathrm{mol} / \mathrm{m}^{3}\right)$

\section{Conclusion}

Scaling analysis has been systematically applied to model equations in obtaining a simplified model. In this analysis, two important difficulties, one in handling nonlinear terms and another in performing trial and error procedure, are addressed. The proposed improvements in the method of scaling analysis results in straight-forward handling of nonlinear terms and avoids trial and error procedure by solving a set of algebraic equations to obtain the scale and reference values. This method is illustrated through several examples of varying complexity. The proposed scaling approach is common to any given model equations with fixed parameters. However, it is to be noted that the proposed procedure may not handle situations when model equations exhibit steady state multiplicity and have dynamic multimode regimes. The $1 \mathrm{D}$ WGS reactor model is used as a case study to obtain scale and reference values, using the proposed scaling analysis. The obtained 
values are verified to be appropriate, as this results in making the dimensionless variables in the 1D model to vary between zero and one. The simplified model obtained using these values is shown to be a good approximation for the detailed model with increased computational efficiency. Thus, from this case study, it is concluded that the proposed scaling analysis is systematic and effective in identifying appropriate scale and reference values, which can be used for any further analysis including simplification of the model equations. Such simplified models can also be used in optimization studies, and further reduction through techniques such as method of characteristics can lead to tractable first principles models that can be used for online control.

\section{Acknowledgement}

The authors gratefully acknowledge support from DOE through grant no. DE-FE0005749 titled "Model-Based Sensor Placement for Component Condition Monitoring and Fault Diagnosis in Fossil Energy Systems".

\section{References}

Balaji, S., Lakshminarayanan, S., Krantz, W. B., 2008. Scaling and sensitivity analysis of a reverse flow reactor. Chemical Engineering Science 63, $342-355$.

Baldea, M., Daoutidis, P., 2007. Dynamics and control of autothermal reactors for the production of hydrogen. Chemical Engineering Science 62, $3218-3230$.

Dahl, J., Weimer, A., Krantz, W., 2004. Sensitivity analysis of the rapid decomposition of methane in an aerosol flow reactor. International Journal of Hydrogen Energy 29, 57 - 65 . 
Kaisare, N., Lee, J., Fedorov, A., 2005. Hydrogen generation in a reverseflow microreactor: 1. Model formulation and scaling. AIChE Journal 51, $2254-2264$.

Kopaygorodsky, E., Guliants, V. V., Krantz, W., 2004. Predictive dynamic model of single-stage ultra-rapid pressure swing adsorption. AIChE Journal 50, 953-962.

Krantz, W., 2007. Scaling analysis in modeling transport and reaction processes: a systematic approach to model building and the art of approximation. John Wiley \& Sons, Inc., Hoboken, New Jersey.

Krantz, W., Lee, H., Chaudhuri, S.R.and Hwang, S.-T., 2012. Nonbuoyancy density-driven convective mass and heat transfer: Scaling analysis and solution methodology. AIChE Journal 58, 678-689.

Mobed, P., Maddala, J., Rengaswamy, R., Bhattacharyya, D., Turton, R., 2014. Data reconciliation and dynamic modeling of a sour water gas shift reactor. Industrial \& Engineering Chemistry Research 53, 19855-19869.

Munusamy, S., Narasimhan, S., Kaisare, N., 2013. Approximate dynamic programming based control of hyperbolic PDE systems using reducedorder models from method of characteristics. Computers and Chemical Engineering 57, 122-132.

Munusamy, S., Narasimhan, S., Kaisare, N., 2014. Order reduction and control of hyperbolic, countercurrent distributed parameter systems using method of characteristics. Chemical Engineering Science 110, 153-163.

Rao, V., Farooq, S., Krantz, W., 2010. Design of a two-step pulsed pressureswing adsorption-based oxygen concentrator. AIChE Journal 56, 354-370. 
Rezvanpour, A., Krantz, W., Wang, C., 2012. Scaling analysis of the electrohydrodynamic atomization EHDA process for pharmaceutical particle fabrication. Chemical Engineering Science 80, 81-90.

Shvartsman, S., Theodoropoulos, C., Rico-MartInez, R., Kevrekidis, I., Titi, E., Mountziaris, T., 2000. Order reduction for nonlinear dynamic models of distributed reacting systems. Journal of Process Control 10, 177-184. 